\title{
The use of wave power plants in water management for combating the greenhouse effect
}

\author{
Antonina Suzdaleva ${ }^{1, *}$, Viktor Beznosov ${ }^{2}$, Valerii Volshanik ${ }^{1}$, and Valentina Kurochkina ${ }^{1}$ \\ ${ }^{1}$ Moscow State University of Civil Engineering, 26, Yaroslavskoye Shosse, 109377,Moscow, Russia \\ ${ }^{2}$ LLC “Alfamed 2000”, 50-55, $15^{\text {th }}$ Parkovaya street, 105523, Moscow, Russia
}

\begin{abstract}
Nowadays, the development of the greenhouse effect is caused not only by industrial facilities emissions into the atmosphere. The contribution of natural and anthropogenic sources of greenhouse gas emissions is becoming increasingly significant. For example, the amount of carbon dioxide produced by the decomposition of organic substances in the zones of degradation of permafrost increases every year. The contribution of the total emission of numerous dispersed sources of atmospheric pollution is also very significant. Traditional methods of control and limitation of greenhouse gas emissions in this case are irrevocable. The solution of the problem is possible only on the basis of the creation of controlled natural and technical systems. The task for the systems is to organize the flow of greenhouse gases from the atmosphere and carbon fixing in the stable components of the environment. The main area of this activity is the stimulation of bioproduction processes. This task is difficult to accomplish in the field of agricultural production. The main objective of the development of this industry is to meet the growing shortage of food products. Against the background of the threat of mass starvation in a number of regions of the world, the problem of the greenhouse effect loses its relevance.
\end{abstract}

\section{The problem of diffuse sources of greenhouse gases}

The use of sea wave energy for power generation is one of the directions of rational and energy-efficient development of water resources. The development of these renewable energy sources, among other things, opens up new ways to preserve the favorable state of the environment in terms of preventing the further development of the observed global climate change. To substantiate the prospects for the use of wave power plants, it is necessary to briefly dwell on the general problems of activities to combat the development of the greenhouse effect.

At the present stage, one of the main difficulties on the way to prevent the development of the greenhouse effect is a large number of heterogeneous sources of receipt (emission) of greenhouse gases into the atmosphere. The main ones are listed below (Suzdaleva, 2016):

\footnotetext{
* Corresponding author: suzdalevaal@yandex.ru
} 
1. Technogenic centralized sources (large industrial and energy facilities);

2. Technogenic diffuse sources (a set of small-scale objects, the functioning of which is accompanied by the emission of harmful substances).

3. Sources of natural greenhouse gas emissions (volcanoes, forest fires)

4. Natural and anthropogenic sources - natural sources, the flow of greenhouse gases from which is provoked by the technogenesis of the environment. For example, the degradation of permafrost (Eldyshev, 2009), accompanied by the emission of huge amounts of carbon dioxide and methane. The warmer the climate becomes, the more intense is the microbiological and chemical oxidation of previously frozen soils. Thus, at the present stage, the process of $\mathrm{CO}_{2}$ emission from the degraded permafrost zones is self-amplifying.

\section{Main methodological approaches}

As the main measures to reduce the rate of development of the greenhouse effect should be considered:

- limiting of greenhouse gas emissions (Fig.1);

- removal of greenhouse gases from the atmosphere, i.e. intensification of greenhouse gas runoff processes (Fig. 2-3).

\section{Greenhouse gas emissions by sector}

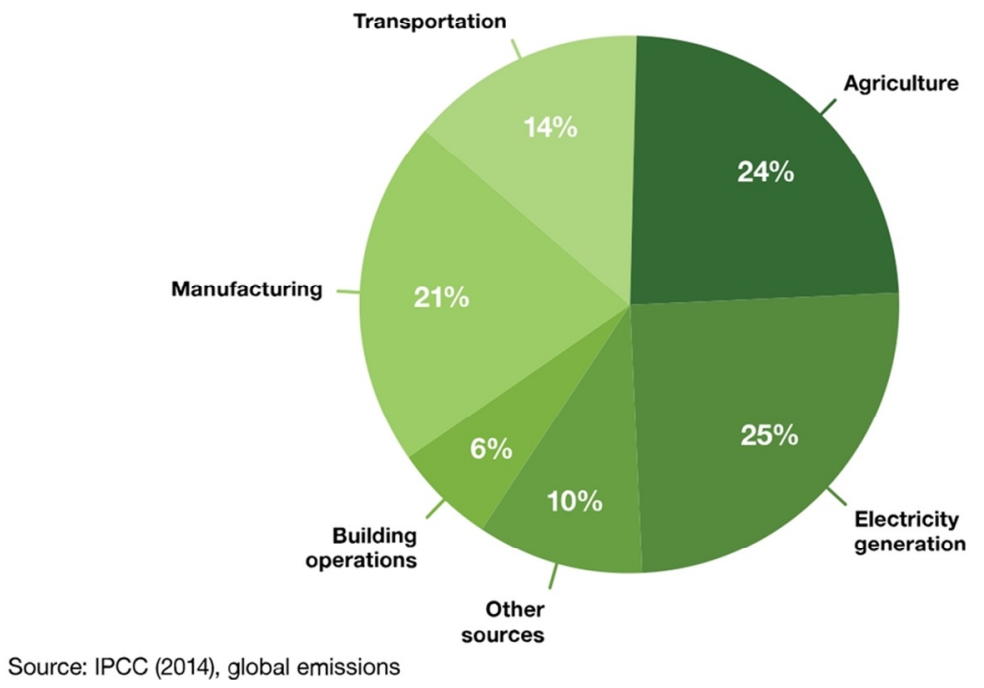

Fig. 1. Greenhouse gas emissions by sector. 


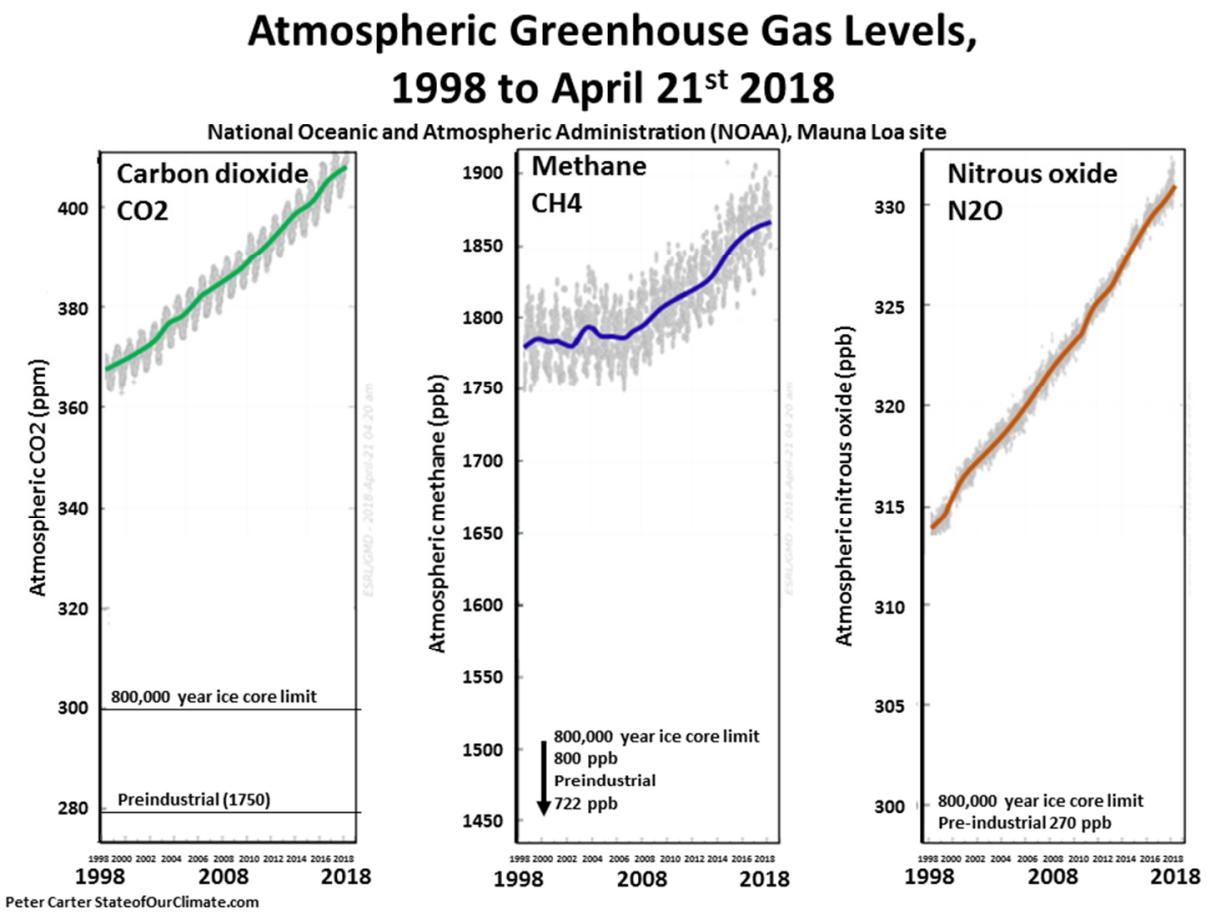

Fig. 2. Atmospheric greenhouse gas level, 1998 to 2018.

Globally averaged greenhouse gas concentrations

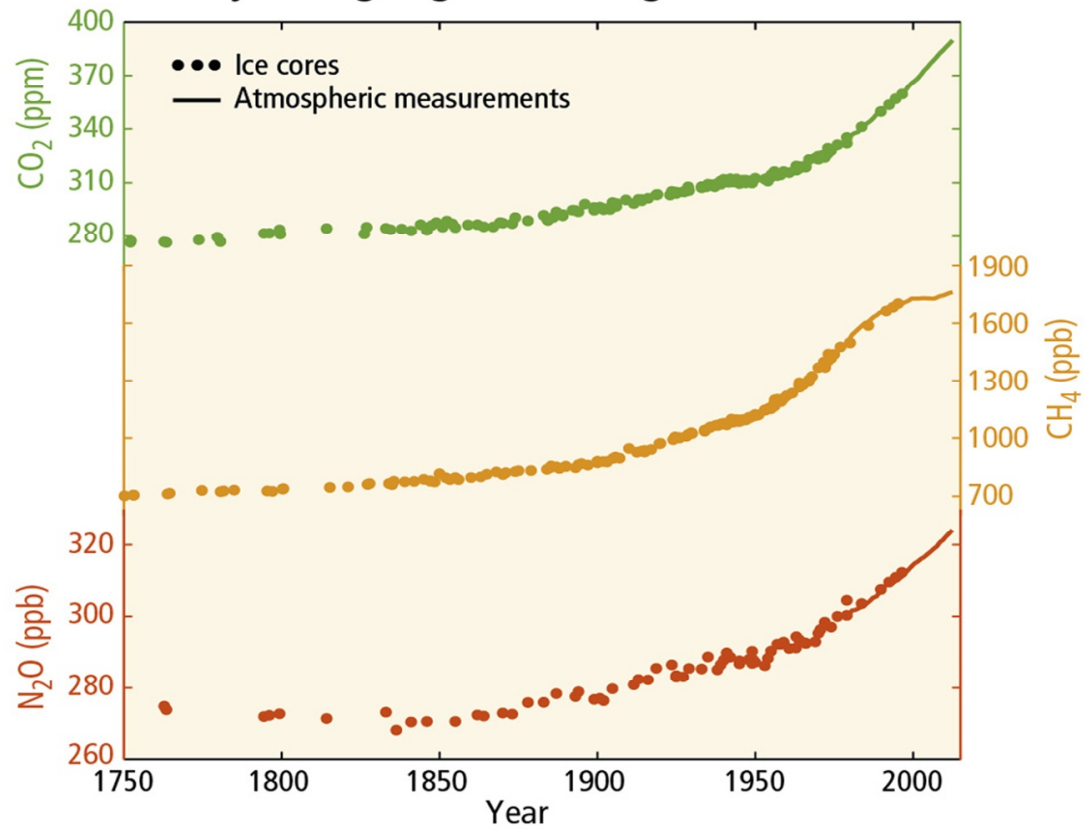

Fig. 3. Globally averaged greenhouse gas contrations. 
Currently, the first of these areas is a priority. The implementation of which is aimed at improving the provisions of the Kyoto protocols and the adoption of national legislation tightening control over greenhouse gas emissions. Importance is also attached to the development of technologies that reduce their emissions level.

Resolution of the problem of the greenhouse effect with restrictive measures is becoming less of a real challenge. These actions can be effective only in relation to anthropogenic centralized and some part of diffuse sources (for example, motor transport). At the same time, population growth and urbanization are accompanied by an increase in the importance of uncontrolled diffuse and natural and anthropogenic sources of greenhouse gas emissions that cause the emergence of "nonpoint source pollution". This problem can be solved only by intensifying the flow of greenhouse gases.

However, it is impossible to create large-scale facilities for air purification from diffuse pollution at the present stage, both for financial and technical reasons. In addition, the operation of such devices will inevitably have a negative impact on avifauna and other biological objects. More promising is the organization of a system of diffusely placed absorbes of greenhouse gases. Such an idea has been repeatedly put forward, but various attempts to implement it have not yet yielded tangible results. The most widespread among such activities was the use of regulated swamp massifs and the formation of green spaces$\mathrm{CO}_{2}$ acceptors, whose products are not intended for food raw materials. The main difficulties along the way were the following:

- the need to allocate large areas and use fertile land to produce plant products that are not used for food purposes (plant-bound carbon must be removed for a long time from the biological cycle, for example, processed into bio-coal);

- high level of expenses for maintenance of these objects (maintenance of water and productive and destructive balance of marsh massifs, watering and fertilizer of plantings, etc.).

It is possible to organize a diffusely placed system of controlled $\mathrm{CO} 2$ runoff, avoiding the above mentioned problems, on the basis of the use of environmentally oriented wave power plants for complex purposes in the water sector. The operation of these devices at the same time can be aimed at performing several tasks that contribute to the improvement of the environmental situation, both globally and locally. The main ones are the following:

- intensification of bioproductivity of macrophyte belt (large-sized algae) of marine coastal areas;

- increase of biodiversity and fishery potential of water areas, as well as increase of stocks of various types of biological resources;

- ecological reclamation of coastal waters due to the inflow of cleaner waters of subsurface layers and increased mobility of the aquatic environment;

- production of environmentally friendly electricity that can be used to process biological products on site.

It should be noted that the growth rates of many mass species of macrophytes and, accordingly, the rate and volume of $\mathrm{CO} 2$ absorption by them, are not inferior to the most productive forms of terrestrial vegetation.

\section{Prospects for the use of wave installations}

The energy potential of wind wave energy is great. The total potential capacity of wind waves of the World ocean is estimated at 30-1000 million MW (Sichkarev, Akulichev, 1989). However, the wave power that can be used by humans is smaller and is estimated at 2.7-5.0 million MW (Volshanik, Zubarev, Frankfurt, 1983). The use of even a small fraction of this energy could cover a significant part of the needs of mankind. However, 
there are a number of reasons that currently do not allow this possibility to be realized. The main ones are the instability of electricity generation over time and the complexity of its transmission to the consumer. However, for use in order to prevent dispersed $\mathrm{CO} 2$ emissions, these disadvantages are not significant, and wave energy has undoubted advantages: geographical distribution and the possibility of placing installations in close proximity to both the macrophyte belt and deep water sources.

The latter circumstance distinguishes wave installations from the majority of so-called "artificial upwelling" devices. The rise of water directly into the surface layer of the sea by them does not always lead to the desired results. Thus, when mixing deep water with surface water, the content of biogens can be reduced to a level that does not have a stimulating effect on the growth of aquatic plants (Beznosov, 2000b), and too low water temperature can cause death and growth retardation of aquatic organisms (Beznosov, Suzdaleva, 2001). In addition, the organization of macrophyte plantations around artificial upwelling devices and their maintenance, require significant costs, they are subject to the destructive effects of storms and create obstacles to navigation.

To date, wave energy devices of various types have been developed and tested in many countries (Volshanik, Zubarev, Frankfurt, 1983). Without stopping at the design solutions used in their creation, we will only point out that almost all of them are applicable to fulfill the goal.

\section{Design principles}

In generalized view, the design includes the following elements: wave power plant, equipped with a pump for lifting deep water; tank - storage of deep water; pipeline, supplying water from the deep horizons to the tank-storage; distribution network in the macrophyte belt.

The type of installation is chosen based on the specific conditions of its operation and the capacity required to cover energy costs. The placement site, on the one hand, is determined by the availability of deep waters with a high bioproductive potential, which can be estimated on the basis of the calculation of potential production according to the Redfield-Richards formula, taking into account the concentrations of phosphates and nitrates in deep waters (Beznosov, 2000b; Suzdaleva and others, 1998/1999). On the other hand, the site should be near the macrophyte belt and, at the same time, have suitable conditions for the operation of power equipment (the level of excitement and its constancy).

In a preliminary assessment of bioproduction potential, the Redfield-Richards stoichiometric model is used. In its simplest form, it is the ratio of the concentrations of the main biogenic elements $(\mathrm{C}: \mathrm{N}: \mathrm{P})$ in the bodies of aquatic organisms. On average, it is equal to $106: 16: 1$. Based on this model, the potential macrophyte production can be calculated (Beznosov, 2000b; Suzdaleva et al., 1998/1999). In the case under consideration, this is the maximum mass of algae that can be formed with the full use of the total stock of biogen in the medium. Under natural conditions, the balance of mineral compounds of phosphorus and nitrogen, as a rule, differs significantly from their ratio in the Redfield-Richards stoichiometric model. Therefore, the calculation of potential production is based on the content of the limiting element, which in deep waters can be both phosphorus and nitrogen. If the ratio of $\mathrm{N} / \mathrm{P}$ concentrations expressed in gram atoms/liter is more than $1 / 16$, then the amount of carbon in the potential production is determined by the amount of phosphorus contained in the water. If this ratio is greater than $1 / 16$, then by the concentration of nitrogen. When assessing the properties of deep waters using this method, it should be remembered that potential production is an abstract indicator suitable only for comparing the bio-production potential of deep waters from different layers. Achievable macrophyte production is much smaller, since all atoms of the limiting biogenic element present in the 
medium cannot be absorbed by plants at once and used to create organic matter during photosynthesis. The actual value of macrophyte production can only be determined experimentally. For example, in experiments conducted in the most favorable environment for algae cultivation, artificially created in special mesocosm containers, it did not exceed $20 \%$ of the potential production (Beznosov, 2000a).

The design of pumping equipment for lifting deep waters is determined by calculating their volume, which contains the amount of biogens required to achieve the planned effect.

The tank-storage is designed to perform two tasks at the same time:

- creation of a reserve of deep waters for periods of long calms, during which the intensity of the operation of wave power plants, and, consequently, the rise of deep waters, significantly decrease or completely stop;

- formation of the optimal temperature regime of deep waters coming to macrophytes.

The solution of the second problem depends on local conditions. In temperate latitudes, it is desirable to increase the temperature of deep waters, so the tank-storage can be placed on the heated surface of the sea. In the tropics, where strong heating of shallow waters and, moreover, the drainage zone negatively affects aquatic organisms, it is desirable to bury the tank-storage. The material from which the storage tank is made must be impervious to sunlight. Otherwise, water bloom may begin in it, and the biogens necessary for the development of macrophytes will be absorbed by phytoplankton.

The location and length of the pipeline supplying the deep water to the storage tank are determined by the hydrological structure of a particular area. The main requirement is the location of the water intake in a layer in which the content of biogens (phosphates and nitrates) would be constantly at a level that ensures high bioproductivity of macrophytes. On the Caucasian coast of the Black sea, where research was conducted artificial upwelling, it was 200-300 m from the shore at a depth of 40-50 m. It should be noted that a good growth of algae was observed in cases when the bottom layers of water contained high concentrations of hydrogen sulfide (Polikarpov and others, 1986). Upon contact with oxygen dissolved in water, hydrogen sulfide rapidly oxidized to sulfur, and deep water lost its toxicity.

The distribution network of water supply is designed for their distribution in the area of the macrophyte belt. It should provide as many plants as possible with biogenes. In its simplest form - it is an extensive network of perforated pipes fixed to the ground among algae. When designing it, it is necessary to take into account the features of the thermal regime and the hydrochemical composition of deep waters, as well as the characteristic features of the hydrodynamics of the coastal zone of the sea at the plant location site. In the temperate and subtropical zones, the optimum temperature for bioproduction processes is a water temperature of $25-30^{\circ} \mathrm{C}$. In the tropics, it can be slightly higher, but not more than $35^{\circ} \mathrm{C}$. At a temperature of $40^{\circ} \mathrm{C}$ or more, almost all types of macrophytes die. An important factor is the mobility of the aquatic environment. The intensity of photosynthesis and the growth rate of algae in surfy areas or areas with strong tidal currents can be 2 times or more higher than that of plants of the same species growing under conditions of low mobility of the aquatic environment. Therefore, forced pumping of water in distribution networks during calm periods can have a significant effect with the help of additional pumps placed in them, operating from batteries recharging during operation of the main power plants during periods of intense wave disturbance. This is especially important in tropical areas, where the heating of shallow water by the sun often reaches an extreme level. Under these conditions, breeding of marine organisms (aquaculture) is possible only through the use of artificial upwelling devices that are used not only to intensify bioproduction processes, but also to cool the waters of the coastal zone of the sea (Beznosov, 2000a). At the same time, the slow advance of deep water in distribution networks (by gravity from storage tanks) may be accompanied by their heating to a critical level. 
Additional pumps in distribution networks can be equipped with devices for bubbling deep waters, i.e. their forced saturation with atmospheric oxygen. This can be done not only for the purpose of oxidizing the hydrogen sulfide contained in them. Often, a significant portion of nitrogen and phosphorus is present in deep waters in the form of organic substances, which are practically not used by macrophytes as a source of biogens. The bubbling of deep waters together with an increase in their temperature contributes to their intensive decomposition with the formation of nitrates and phosphates necessary for plants.

\section{Assessment of greenhouse gas absorption efficiency}

The results of experimental studies conducted on the Black sea (in the area of Anapa city), allow us to assess the potential of wave installations as tools that ensure the flow of carbon dioxide from the atmosphere. The rise of deep waters from 40-60 m caused an increase in algae biomass by more than 2 times, based on the duration of the growing season (Beznosov, 2000a). The average mass of the macrophyte belt in rocky shallow waters (up to a width of $10 \mathrm{~m}$ ) per 1 linear meter of coastline in this area was $135 \mathrm{~kg}$, which corresponded to the average value of this parameter in other areas of the Black sea (Kalugina-Gutnik, 1975). Raw algae contain an average of $10-30 \%$ solids and $70-90 \%$ water (Kizeveter and others, 1981). The carbon content of different types of macrophytes varies widely from 26.7 to $56.3 \%$ by weight of dry matter (Terrados, 1995; Bogolitsyn end others, 2012). Taking the average values of these indicators (dry matter content- $20 \%$, the share of carbon- $42 \%$ ), according to approximate calculations, it was found that on a one kilometer section of the coastal zone of macrophytes (10 m wide) with the use of complex wave power plants, 11.340 tons of carbon can be deposited in addition, which in terms of $\mathrm{CO}_{2}$ is more than 40 tons.

For comparison, the annual $\mathrm{CO}_{2}$ emission from the same area (1 ha) of thawing permafrost soils is 3.2-3.7 tons, which is more than 10 times less (Kononov, 2006).

The results obtained should not be considered as limiting possibilities of the proposed technology of $\mathrm{CO}_{2}$ runoff from the atmosphere, since deep waters with a high content of biogens can be delivered to many coastal waters. In addition, technical aquaculture can be used to improve efficiency. For example, to obtain algae mass in large volumes, it was proposed to grow a giant and extremely productive brown Alga macrocystis, also called giant kelp (laminaria) (Wilcox, 1982). Some specimens of this type of macrophyte reach a length of more than $45 \mathrm{~m}$ and can grow at a speed of $60 \mathrm{~cm}$ every day.

\section{The removal of biomass and carbon sequestration}

It is obvious that in order to achieve a long-term result to regulate the development of the greenhouse effect, the mass of algae obtained by enriching coastal waters with biogens must be removed from the marine environment and disposed in a proper way. It should be noted that the collection and disposal of additionally grown algal mass is not only a prerequisite for the use of wave power plants to combat the development of the greenhouse effect. Ignoring this aspect not only precludes the achievement of any positive results, but also naturally leads to very undesirable consequences. An uncontrolled increase in the intensity of bioproduction processes in areas of artificial upwelling, for which the special term "destratification eutrophication" was proposed (Beznosov, 2000a), entails the same environmental consequences as other forms of anthropogenic eutrophication of water bodies. For example, in the Anapa Bay (Anapskaya Bukhta), where algae mass was not collected, an outbreak of macrophyte development at the beginning of the summer period, caused by the ingestion of biogens with effluents from the coastal territory, in the 
subsequent period caused a sharp deterioration in the ecological situation as a result of decomposition of plant debris and secondary pollution of coastal waters (Goryunova Beznosov, 2004).

Collection of algae mass can be carried out in two main ways-cleaning storm emissions and collecting growing macrophytes. To mechanize the latter, a number of technologies are used: leveling the relief of rocky shallow waters; formation of macrophyte thickets on artificial reefs, etc.

When collecting algal mass and its subsequent disposal, it is necessary to take into account some environmental aspects of this activity that are not directly related to the sphere of combating the development of the greenhouse effect. Macrophytes are one of the most common means of bioremediation, i.e. they are used to extract various pollutants from the environment. They are able to accumulate significant amounts of various substances, the removal of which from water improves its quality. This effect is widely used in biomelioration of various water bodies. In heavily polluted coastal waters, this process can include two stages or more. An example is the successive change of remediation and sanitary aquaculture of macrophytes. At the first of these stages (remediation), artificial cultivation of species capable of mass development in heavily contaminated areas is carried out, improving their condition to a certain level. After an improvement in the ecological situation, such forms as, for example, ulva green algae in the Black Sea give way to other macrophyte species, for example, brown algae from the cystosira genus, controlled growth of which is able to maintain a favorable ecological situation under high anthropogenic load on the water area (sanitary aquaculture). Field experiments conducted in the most polluted areas of the Anapa Bay showed that the use of artificial upwelling devices significantly accelerated the process of water improvement using macrophytes, which was almost always accompanied by the formation of significant volumes of algal mass, and, consequently, additional fixation of atmospheric carbon. The rise of deep waters allowed the remediation of green algae to begin in areas where extremely high levels of oil and household pollution made it difficult to carry out such activities. At the same time, the change in the mass species of algae forming the macrophyte coastal belt required adjustments to the technology for collecting algae mass.

It should also be noted that the macrophyte belt and their coastal wash-outs are the habitat of various aquatic organisms. Therefore, the planning of the volumes and timing of the removal of algal mass should be based on the results of special studies.

For disposal of excess or contaminated mass of macrophytes, in the context of the considered problem, it is necessary to recommend the methods designated by the abbreviation CCS (Carbon Capture and Storage-carbon capture and storage) (Eldyshev, 2009). A number of projects propose the introduction of these methods for the production of bio-coal. In this case, the abbreviation BECCS (Bio-energy with carbon capture and storage) is also used to refer to them (Kokorin, 2014). Since the main substance artificially removed from the atmosphere is $\mathrm{CO} 2$, the technologies used for this purpose are also designated by the term "carbon dioxide sequestration technologies" (Cherepovitsyn, 2013). The resulting products are subjected to compression and under high pressure are transported through pipelines to the burial sites. As the latter, it is proposed to use various voids formed in the bowels of the Earth as a result of mining, which are widely used now, for example, for the disposal of waste from oil companies (drilling waste).

\section{Conclusion}

Summarizing the presented materials, it can be argued that the use of complex wave power plants is both an effective way to combat nonpoint atmospheric pollution with carbon dioxide, and a method of multifaceted improvement of the environmental situation in the 
water sector. The operation of wave installations in coastal and marine waters with a wellthought-out organization is accompanied by purification of the aquatic environment from pollutants, protects the organisms of shallow waters from extreme warming in the summer. These factors contribute to the conservation of biodiversity and increase marine biological resources. Thus, the use of complex wave power plants forms local controlled natural and anthropogenic technical systems (Suzdaleva, 2016). Favorable conditions for the existence of organisms are regulated in them by the constant operation of technical devices that provide pumping and distribution of deep water in macrophytes belt. This method is also applicable to large reservoirs and lakes, whose bottom water masses contain a significant amount of biogens, and in the coastal zone a macrophyte belt is developed.

\section{References}

1. V.N. Beznosov, Ecological consequences of the violation of the stratification of the se. Dr. biol. sci. diss. (MSU, Moscow, 2000)

2. V.N. Beznosov, Oceanology 40(6), 890-894 (2000)

3. V.N. Beznosov, A.L. Suzdaleva, Oceanology 41(6), 870-873 (2001)

4. K.G. Bogolitsyn, P.A. Kaplitsin, N.V. Ul'yanovskii, O.A. Pronina, Khimiya rastitel'nogo syr'ya 4, 153-160 (2012)

5. V.V. Volshanik, V.V. Zubarev, M.O. Frankfurt, Use of wind energy, ocean waves and currents (VINITI, Moscow, 1983)

6. S.V. Goryunova, V.N. Beznosov, Aktual'nye problemy ekologii i prirodopol'zovaniya, RUDN 5(2), 114-122 (2004)

7. Yu.N. Eldyshev, Ekologiya i zhizn' 9(94), 48-56 (2010)

8. A.A. Kalugina-Gutnik, Phytobenthos of the Black Sea (Nauk. Dumka, Kiev, 1975)

9. I.V. Kizeveter, M.V. Sukhoveeva, L.P. Shmel'kova, Commercial algae and grass of the Far Eastern seas (Legkaya i pishchevaya promyshlennost', Moscow, 1981)

10. A.O. Kokorin, Climate Change: Review of the IPCC Fifth Assessment Report (Vsemirnyi fond dikoi prirody, Moscow, 2014)

11. A.V. Kononov, Emission of carbon dioxide by cryogenic soils of larch forests in Central Yakutia depending on hydrothermal conditions. Cand. biol. sci. diss. (Yakutsk, 2006)

12. G.G. Polikarpov, G.E. Lazorenko, N.N. Tereshchenko, Xenobiotic and biogenic properties of the aquatic environment from the Red Sea zone of the Black Sea for algae, Dokl. AN USSR 4, 76-79 (1986)

13. V.I. Sichkarev, V.A. Akulichev, Wave power stations in the ocean (Nauka, Moscow, 1989)

14. A.L. Suzdaleva, Creation of managed natural-technical systems (ID ENERGIYA, Moscow, 2016)

15. A.L. Suzdaleva, V.N. Beznosov, S.V. Goryunova, B.P. Pshenichnyi, Vestnik RUDN, Ser. Ekologiya i bezopasnost' zhiznedeyatel'nosti 3, 52-57 (1998/1999)

16. A.E. Cherepovitsyn, K.I. Sidorova, N.V. Smirnova, Neftegazovoe delo 5, 459-473 (2013)

17. J. Terrados, Scientia Marina 59(1), 49-56 (1995)

18. H.A. Wilcox, Experimentia 38(1), 31-35 (1982) 